\title{
Modeling of machined depth in laser surface texturing of medical needles
}

\author{
Xingsheng Wang ${ }^{1,2}$, Peidong Han $^{2}$, Marco Giovannini ${ }^{2}$, Kornel Ehmann ${ }^{2}$
}

\begin{abstract}
The present paper addresses the experimental modeling of process parameters in laser surface texturing (LST) of medical needles. First, experiments were carried out based on Taguchi methodology. The laser process parameters considered during LST were the circumferential overlap, axial overlap and the overscan number. A second-order regression model of the machined depth for LST was developed based on the experimental results. Second, a predictive model for the machined depth based on least squares support vector machines (LS-SVM) with radial basis functions was constructed using the same experimental swatches. Grid search and leave-one-out cross-validation were used to determine the optimal parameters of the LS-SVM model. The comparison between the second-order regression model and the LS-SVM model was carried out. The experiments indicated that the LS-SVM model is capable of better predictions of the machined depth than the second-order regression model. The validity of the LS-SVM model has been checked through the creation of micro-channels with blended edges. It was found that the predicted profile was in a good agreement with the experimental profiles. The LS-SVM model can be used to predict machined geometry of the micro-channels on medical needles.
\end{abstract}

Keywords Laser surface texturing; Medical needles; LS-SVM; Predictive model of machined depth;

\section{Introduction}

Medical needles are the most commonly used medical devices and are widely used in various minimally invasive percutaneous procedures like tissue biopsy, regional anesthesia, blood sampling, brachytherapy, catheter insertion, abscess drainage and deep brain biopsy [1-2]. The positioning accuracy of the medical needle is of critical importance for the success of these procedures. A practical approach to improve the positioning accuracy is to use ultrasound to guide the placement of echogenic needles during needle insertion [3]. Micro-dimples, micro-corner reflectors, and micro-channels are often created near the needle tip to serve as ultrasound wave reflectors [3-6].

Echogenic needles increase their visibility under sound guided percutaneous procedures; however, soft materials tend to conform to the profile of the micro-features on the needles resulting in increased friction between the needle and soft tissue. Higher insertion forces may lead to patient discomfort and target movement resulting in placement errors. Han et al. suggested that using micro-features with blended edges, instead of sharp edges, on echogenic needles can reduce the edge effect and reduce the 
insertion force [7].

At present, micro-features can be machined by micro mechanical cutting [8], micro-EDM [9-10], micro-ECM [10-11], micro-ultrasonic machining [12], ion beam machining [13-14], laser beam machining [15-16] as well as hybrid micro manufacturing processes such as vibration assisted micro cutting [17], laser assisted micro cutting [18] and ultrasonic assisted EDM [19]. Among these technologies, the ultra-short laser pulsed laser ablation process is the front-runner, and widely used in precision machining micro-features in various materials. Micro-channels are versatile micro-features that are often used in surface texturing and can be arrayed or joined to form patterns and free-form geometries. In this paper, laser surface texturing (LST) is used to manufacture micro-channels on needles. The final geometry of the machined features depends on numerous laser process parameters such as average power, peak power, repetition rate, wavelength, etc. In order to ensure that the desired geometry of micro-features, especially with blended edges, is achieved through LST, the laser process parameters must be reasonably selected. An effective way to select appropriate process parameters is to develop predictive models for the machined geometry.

A number of studies have developed models for predicting the machined geometry or depth during laser beam machining processes. Existing predictive models can be broadly divided into three categories: analytical solution-based, numerical solution-based and experiment-based models. Jiang et al. [20] predicted the depth of grooves in sheet metal cutting with pulsed Nd:YAG laser using an analytical model. A lumped parameter model for relating cutting parameters and material properties to cut depth has been developed by Li et al. [21]. This model can be applied to a wide set of parameters ranging from low to high laser powers and slow to fast cutting speeds. Modeling based on analytical solutions is usually centered on some assumptions and is not always able to solve the whole system in practical cases. Kim [22-23] developed two numerical models with boundary element methods which considered an unsteady heat-transfer phenomenon in evaporative laser cutting to predict groove shape. Compared to analytical models, numerical modeling is usually based on fewer assumptions but needs more computing power. Yousel et al. [24] used multi-layered neural network to develop a predictive model for the nonlinear laser cutting process. The predicted variables were groove depth and diameter. Tsai et al. [25] developed an artificial neural network (ANN) model which involves more laser process parameters such as current, frequency and cutting speed to predict the depth of cut, while Ciurana et al. [26] developed an ANN model to study the relation between process parameters and T-shaped deep features in pulsed laser micromachining of hardened AISI H13 steel. The training of artificial neural network models is based on abundant experimental data and proper algorithms which determine the accuracy of the developed models. Hybrid methods also exist that combine two or more methods to achieve proper results. Davis et al. [27] created a mathematical model based on baseline experiments to evaluate the effect of pulse overlap on laser micro machined geometry. The model generated three-dimensional geometry prediction plots based on the input parameters, including desired pulse overlap in the $X$ - and $Y$-directions, width and length of the channel, beam diameter, and a set of two baseline data points relating the number of pulses and ablated depth at the controlled laser operating parameter levels. Gilbert et al. [28] presented a mathematical model to determine the micro-topography of the pulsed laser ablated surface. The model used an experimentally determined logarithmic relationship between fluence levels and the maximum depth of the laser crater while taking into account the overlapping of pulses, depth of field and the surface gradient of the component. 
In spite of the fact that LST has been used to create some micro features on medical needles, no studies to date have been carried out to develop a model to predict the geometry of the machined micro features. An accurate prediction of the effect of various laser process parameters will allow the desired geometry on the needles to be achieved through LST. Developing a predictive model through experiments is an effective way and has been widely used in laser beam machining. The key to a successful predictive model, based on experiments, is the selection of an appropriate model and its parameters.

In this paper, two different approaches to construct predictive models that relate the machined depth to three laser process parameters, i.e., the circumferential overlap, axial overlap and overscan number, in LST of medical needles will be used. For this purpose, an experimental setup for LST is developed and used to create micro-channels on needles, as well as to obtain experimental data. The first approach is a second-order regression model based on the Taguchi method, while the second approach is modeling by means of least squares support vector machines (LS-SVM). This study compares the performance of these two different modeling methodologies. Micro-channels with blended edges are manufactured to present a case study to test the performance of the predictive models.

\section{Experimentation}

\subsection{Experimental setup}

A commercial diode-pumped Nd: YVO4 laser (Lumera laser, RAPID) with a pulse duration of 8 ps operating at its second harmonic (532 nm wavelength) was used for LST of medical needles. The pulse repetition rate can be adjusted from 10 to $500 \mathrm{kHz}$. Laser pulses can be selected by TTL-trigger using external TTL pulses, which allows repetition rate to be coordinated with machining trajectory to optimize the placement of each laser pulse. The principle of pulse picking is shown in Fig. 1. The beam was linearly polarized with a $0.6 \mathrm{~mm}$ diameter and its profile was Gaussian (TEM00). The Gaussian beam was brought to a $25 \mathrm{~mm}$ trip-element focusing lens through a fully enclosed beam delivery system comprising a beam expender and an array of turning mirrors. Through the focusing lens, a diffraction limited spot size of $10 \mu \mathrm{m}$ was realized at the focal point. The laser machine was equipped with a 5-axis motion stage (Aerotech Inc.) with a translation resolution of $10 \mathrm{~nm}$ and rotational resolution of $0.00001 \mathrm{deg}$.

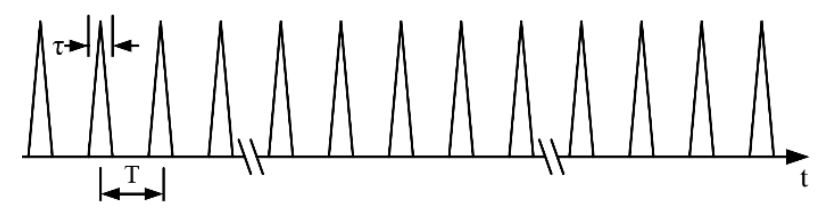

(a)

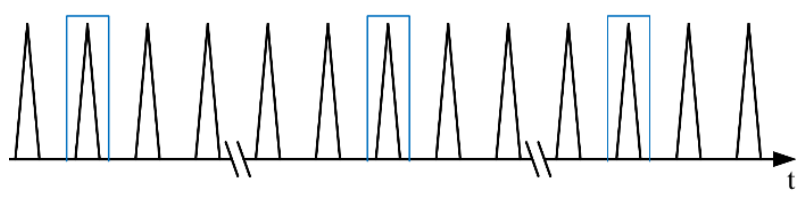

(b) 


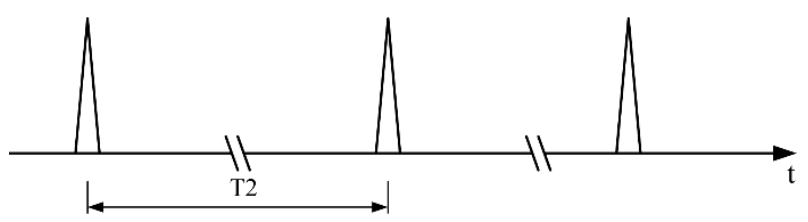

(c)

Fig. 1 Amplitude versus time of laser pulse trains: (a) generated pulse train with pulse separation (T) and pulse duration $(\tau)$, (b) pulses are 'picked', (c) desired pulse separation is achieved (T2)

Figure 2 shows a schematic of the experimental setup for LST of medical needles. The needle was mounted on the needle fixture and alignment subsystem consisting of a micrometer adjuster, a set of ER8 collets and several adapters. The resolution of the micrometer adjuster is $10 \mu \mathrm{m}$, which provides an accuracy of approximately $10 \mu \mathrm{m}$ of the needle fixture and alignment subsystem. A focusing and vision subsystem comprising of a polarizing beam splitter, a polarization-insensitive beam splitter, a laser focusing lens, a fiber optical illuminator, a CCD camera, and a notch filter was used to assist in focusing and to monitor the machining process. The focal position of the CCD camera was aligned with the laser focus within an accuracy of $\pm 10.0 \mu \mathrm{m}$ in the $Z$ direction. Focusing of the laser on a needle was achieved through focusing the camera on the needle.

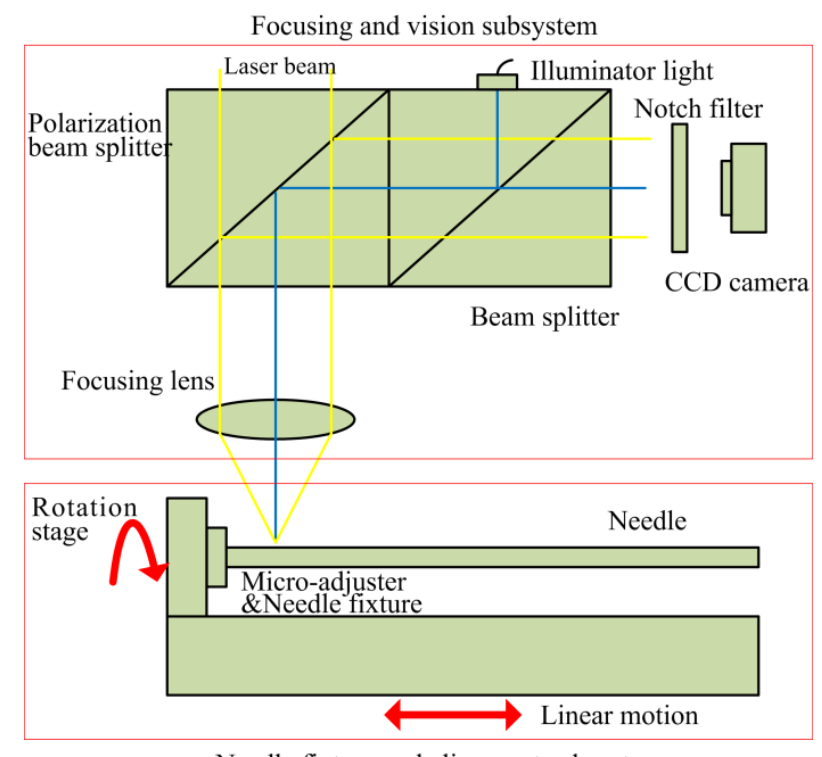

Needle fixture and alignment subsystem

Fig. 2 Schematic of the laser surface texturing setup

\subsection{Experimental plan}

Figure 3 presents the schematic for the generation of a typical micro-channel on medical needles through overlapping craters which are machined on the workpiece surface by a single laser pulse. As the figure indicates, the degree of overlapping along the circumferential direction determines the depth and width of the single-channel. Single-channels can be overlapped along the axial direction to create a multi-pass micro-channel that can be subsequently overscanned in layers to generate the final desired 3D micro-channel.

The micro-channel dimensions depend on the laser machining parameters. Hence, the selection of 
ranges of various parameters is an important issue for the LST of medical needles. The two main factors in LST are the circumferential overlap and the axial overlap, which determine a final geometry of the laser machined surface. Throughout this paper, the circumferential overlap is defined as the percentage of diameter overlap for successive pulses in the circumferential direction, while the axial overlap is defined as the percentage of diameter overlap between subsequent single-channels in the axial direction. The relationship between the circumferential overlap with other parameters is as follows:

$$
C O_{\mathrm{p}}=1-\frac{v_{\mathrm{rot}} \times \pi \times D}{60 \times d \times P F}
$$

where $C O_{\mathrm{P}}$ is the circumferential overlap, $v_{\text {rot }}, D, d$ and $P F$ represent the needle rotational speed (rpm), needle diameter $(\mathrm{mm})$, laser spot diameter $(\mathrm{mm})$ and pulse frequency $(\mathrm{Hz})$, respectively. According to Eq. (1), it is obvious that the circumferential overlap depends on the needle rotational speed and pulse frequency. Hence, the circumferential overlap can be varied by two methods, i.e.: (i) by changing the needle rotational speed while the pulse frequency is kept constant and (ii) by changing the pulse frequency while the needle rotational speed is kept constant.

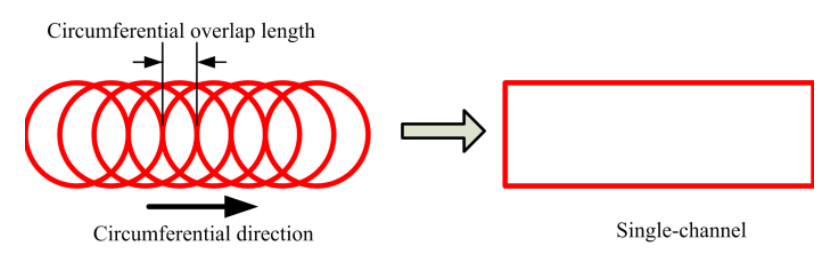

(a)

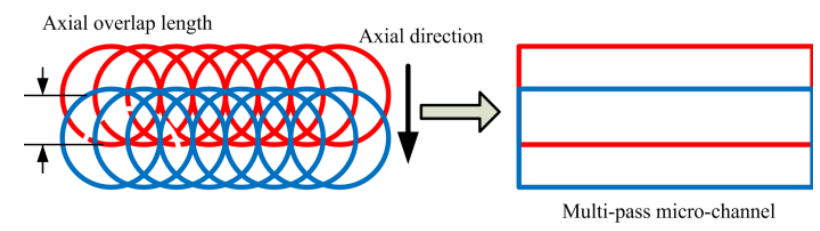

(b)

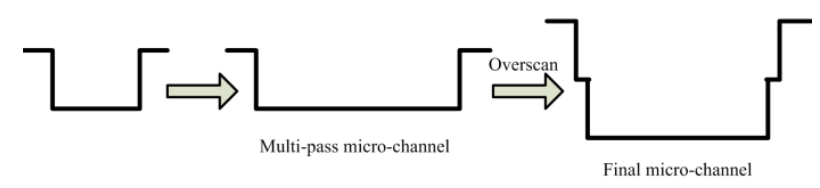

(c)

Fig. 3 Schematics for the formation of: (a) Single-channel through circumferential overlapping, (b) Micro-channel through axial overlapping, (c) 3D micro-channel through overscanning

Circumferential channels without overlapping in the axial direction were machined to study the effect of the variation of the circumferential overlap by two different methods on the machined depth. A solid bevel tip needle with a diameter of $1.8 \mathrm{~mm}$ was used as the workpiece for LST that was made of AISI 304 stainless steel. The operating frequency was set to $100 \mathrm{kHz}$ at an average power of $0.6 \mathrm{~W}$, yielding a pulse energy of approximately $3 \mu \mathrm{J}$ after reflection and transmission losses within the beam delivery system, as measured by an external power meter (GentecSolo2). Through the motion program 
combined with TTL-trigger, the desired pulse frequency was obtained by selectively outputting pulses from the $100 \mathrm{kHz}$ pulse train. The focal plane of the laser was set at the surface of the needle and remained unchanged during the texturing process. The range of the circumferential overlap, rotation speed and pulse frequency has been selected after performing some prior experiments. The machining trajectory was repeated 5 times to achieve a depth that was easily measurable.

For the results shown in Fig. 4(a), the needle rotational speed was kept constant at 5, 10, $15 \mathrm{rpm}$, respectively, and the circumferential overlap was changed by varying the pulse frequency. As it can be observed, an increase in the circumferential overlap increases the machined depth. In this case, with the same circumferential overlap the needle rotational speed has no significant effect on the machined depth. Figure 4(b) illustrates the experimental results when the variation of the circumferential overlap was achieved by varying the needle rotational speed while the pulse frequency was kept constant at 200 , $400,600 \mathrm{~Hz}$, respectively. It also reveals that the machined depth increases with the circumferential overlap. Similarly, the pulse frequency has no obvious influence on the machined depth with the same circumferential overlap.

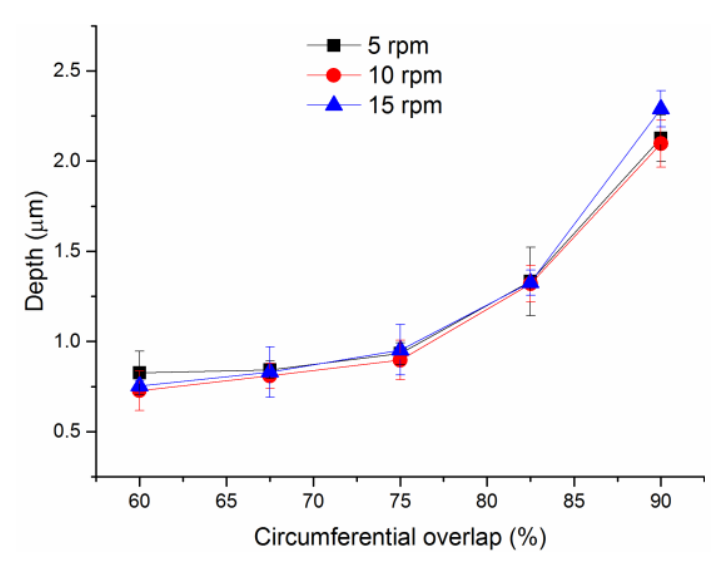

(a)

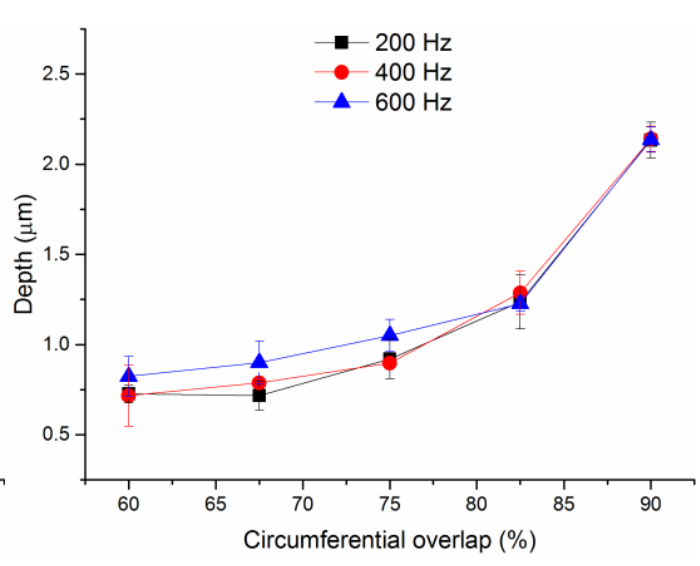

(b)

Fig. 4 Effect of circumferential overlap on machiend depth by changing (a) pulse frequency, (b) needle rotation speed

In this investigation, the circumferential overlap was altered by changing the pulse frequency because of its easy implementation. Axial overlap was achieved by shifting the needle by a certain distance along the axial direction. Also, for a constant setting of the circumferential overlap and axial overlap, the overscan number has a significant influence on the final geometry formed. The present experimental study attempts to develop a model to predict the final geometry of the machined micro-channels based on these two overlap factors and the overscan number.

\subsection{Taguchi methodology based experimental design}

Regular micro-channels with a length of $450 \mu \mathrm{m}$ and a width of $50 \mu \mathrm{m}$ were chosen to be machined. Figure 5 shows the schematic of the strategy for machining regular micro-channels. As it can be seen in the figure, a single-channel was initially machined with a certain circumferential overlap. Then, the needle was shifted by a small distance depending on the axial overlap along the axial direction. Subsequently, another single-channel was machined in the inverse direction at the same circumferential overlap as for the first single-channel. This was repeated until the desired width of the micro-channel was achieved. The machining trajectory was repeated to obtain different depths. An L25 
orthogonal array based on the Taguchi method was used for designing the experiments. The circumferential overlap, axial overlap and overscan number were selected as the variable parameters. The range of these laser process parameters has been selected based on pilot experiments. The parameters used in the experiment are shown in Table 1. The other process parameters were the same as mentioned previously.

Table 1 Laser process parameters and levels used

\begin{tabular}{|c|c|c|c|c|c|c|c|}
\hline Symbol & Factors & Unit & Level 1 & Level 2 & Level 3 & Level 4 & Level 5 \\
\hline A & $\begin{array}{c}\text { Circumferential } \\
\text { overlap }\end{array}$ & $\%$ & 60 & 67.5 & 75 & 82.5 & 90 \\
\hline B & Axial overlap & $\%$ & 60 & 67.5 & 75 & 82.5 & 90 \\
\hline $\mathrm{C}$ & Overscan number & & 1 & 3 & 5 & 7 & 9 \\
\hline
\end{tabular}

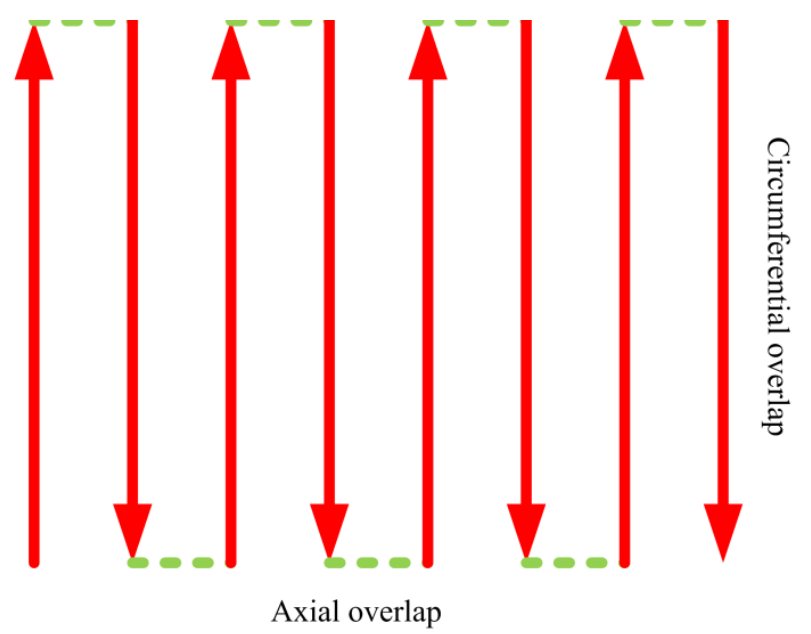

Fig. 5 Schematic of the machining of regular micro-channels

\subsection{Experimental results}

The generated micro-channels were characterized by an Alicona Infinite Focus Microscope (IFM) following a thorough ultrasonic cleaning to eliminate the debris from the textured surfaces. The depth of the micro-channels was measured at three different cross sections and averaged. The experimental measured depths are listed in Table 2. Figure 6 shows the image of machined channels for experiments No.6 to No.10 listed in Table 2. 


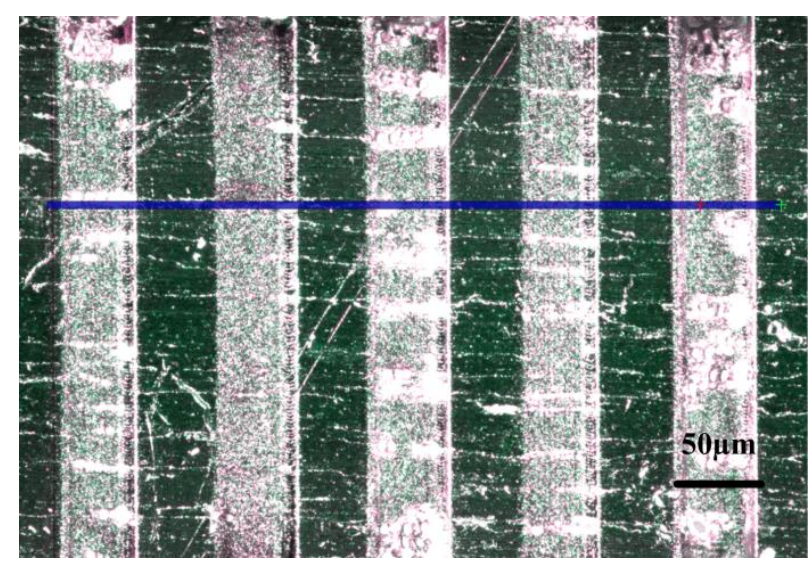

(a)

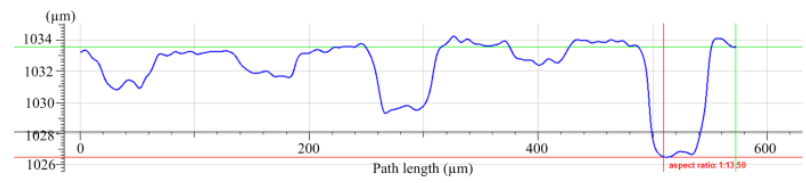

(b)

Fig. 6 Micro-channel results (a) surface image, (b)cross-section profiles

Table 2 Experimental data set

\begin{tabular}{ccccc}
\hline Exp no. & A $(\%)$ & B $(\%)$ & C & Depth $(\mu \mathrm{m})$ \\
\hline 1 & 60 & 90 & 9 & 10.75 \\
2 & 60 & 67.5 & 3 & 1.37 \\
3 & 60 & 82.5 & 7 & 5.21 \\
4 & 60 & 75 & 5 & 2.65 \\
5 & 60 & 60 & 1 & 0.51 \\
6 & 67.5 & 67.5 & 5 & 2.76 \\
7 & 67.5 & 90 & 1 & 1.47 \\
8 & 67.5 & 75 & 7 & 4.12 \\
9 & 67.5 & 60 & 3 & 1.26 \\
10 & 67.5 & 82.5 & 9 & 7.68 \\
11 & 75 & 90 & 3 & 5.01 \\
12 & 75 & 67.5 & 7 & 3.76 \\
13 & 75 & 60 & 5 & 2.35 \\
14 & 75 & 82.5 & 1 & 1.13 \\
15 & 75 & 75 & 9 & 6.48 \\
16 & 82.5 & 82.5 & 3 & 4.26 \\
17 & 82.5 & 60 & 7 & 4.35 \\
18 & 82.5 & 75 & 1 & 1.04 \\
19 & 82.5 & 90 & 5 & 11.80 \\
20 & 82.5 & 67.5 & 9 & 6.21 \\
21 & 90 & 67.5 & 1 & 1.46 \\
22 & 90 & 82.5 & 5 & 11.63 \\
23 & 90 & 75 & 3 & 4.39 \\
24 & 90 & 90 & 7 & 23.15 \\
\hline
\end{tabular}




\section{Modeling of machined depth using second-order regression}

Response surface regression was used for establishing the relation between the machined depth and LST parameters. A general second-order polynomial was utilized for the response surface regression expressed as follows[29]:

$$
y=\beta_{0}+\sum_{i=1}^{n} \beta_{i} x_{i}+\sum_{i=1}^{n} \beta_{i i} x_{i}^{2}+\sum_{\substack{i, j=1 \\ i \neq j}}^{n} \beta_{i j} x_{i} x_{j}+\varepsilon
$$

where $y$ is the machined depth, and $x_{i}$ are the coded values of the $i^{\text {th }}$ laser process parameters. $\beta_{0}, \beta_{i}, \beta_{i i}$, and $\beta_{i j}$ are regression coefficients to be estimated and $\varepsilon$ is the experimental error.

The step-wise regression method, which automatically eliminates the insignificant model items was applied using the Design Expert software. The following regression model was determined:

$$
\text { Depth }=202.48-226.22 A-316.87 B-0.13 C+
$$

$$
158.37 A B+1.68 B C+111.51 A^{2}+141.87 B^{2}
$$

where $A, B$ and $C$ represent the circumferential overlap, axial overlap and overscan number, respectively.

To test the performance of the developed model, three error functions were used, i.e., absolute fraction of variance $R^{2}$, mean absolute percent error $e_{\mathrm{M}}$ and root mean square error $e_{\mathrm{R}}$ [30]:

$$
\begin{aligned}
& R^{2}=1-\sum_{i=1}^{N}\left(\frac{m-o}{o}\right)^{2} \\
& e_{\mathrm{M}}=\frac{1}{N} \sum_{i=1}^{N}\left|\frac{m-o}{o}\right| \\
& e_{\mathrm{R}}=\sqrt{\frac{1}{N} \sum_{i=1}^{N}\left(\frac{m-o}{o}\right)^{2}}
\end{aligned}
$$

where $N$ is the number of samples, $m$ and $o$ are the experimental and predictive values, respectively.

The comparison between the second-order regression model and the experimental results is shown in Fig. 7. For the second-order regression model, $R^{2}$ is $0.9989, e_{\mathrm{R}}$ is $59.26 \%$ and $e_{\mathrm{M}}$ is $31.22 \%$, respectively. As shown in Fig. 7, the regression model can predict, to some extent, the machined depth reasonably well. However, the training error of the regression model, e.g., $e_{\mathrm{R}}$ and $e_{\mathrm{M}}$ are still large, and a better predictive model is needed. 


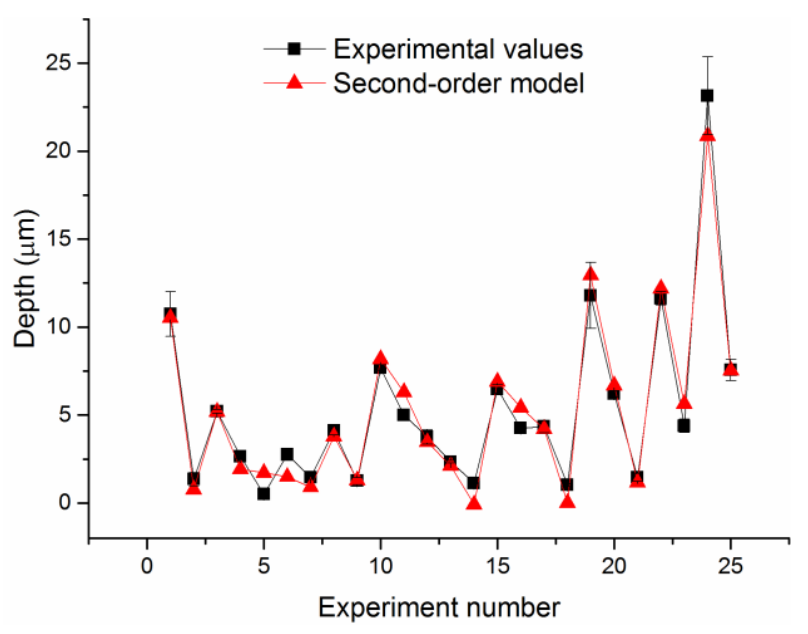

Fig. 7 Comparison of experimental values and training results of regression model

\section{Modeling of machined depth using LS-SVM}

SVM is a relatively novel machine-learning tool and a powerful methodological approach to solve problems in nonlinear classification or regression. It has apparent advantages in classifying and predicting with small sample sizes. LS-SVM was introduced by Suykens et al. [31]. It only requires the solution of a set of linear equations instead of the long and computationally difficult quadratic programming problem involved in standard SVM. It is computationally more efficient than the standard SVM method. LS-SVM has recently been successfully used in predictive models in the field of manufacturing [30, 32-33].

\subsection{Principles of LS-SVM}

For a given training dataset of $N$ data points $\left\{\left(\boldsymbol{x}_{i}, \boldsymbol{y}_{i}\right), i=1,2, \ldots, N\right\}$, where $\boldsymbol{x}_{i} \in \boldsymbol{R}^{n}$ is a $n$-dimensional input data and $\boldsymbol{y}_{i} \in \boldsymbol{R}$ represents the output data. For nonlinear regression, a nonlinear mapping function $\varphi(\cdot)$ is applied to map the original input to a higher dimensional feature space. Then, LS-SVM constructs a linear model in this high dimensional feature space as follows [33]:

$\boldsymbol{y}=\boldsymbol{w}^{T} \varphi(\boldsymbol{x})+b$

where $\boldsymbol{w}$ is the weight vector and $b$ is the bias term. Thus nonlinear function estimation in original space becomes a linear function estimation in feature space.

LS-SVM involves equality constraints instead of inequality constraints by using a least squares cost function [32]. The optimization problem and equality constraints are formulated as:

$\min J(\boldsymbol{w}, \boldsymbol{e})=\frac{1}{2} \boldsymbol{w}^{\mathrm{T}} \boldsymbol{w}+\gamma \sum_{i=1}^{N} e_{i}^{2}$

subject to:

$\boldsymbol{y}_{i}=\boldsymbol{w}^{T} \varphi\left(\boldsymbol{x}_{i}\right)+b+e_{i} \quad i=1,2, \cdots N$ 
where $\gamma$ is a regularization parameter in optimizing the trade-off between minimizing the complexity of the model and minimizing the training errors, and $e$ is the random error between the actual output and the desired output.

The task of finding the optimal parameters is to minimize the prediction error of the regression model described in Eq. 8. In order to solve this optimization problem, a Lagrange function is constructed as:

$$
L(\boldsymbol{w}, b, \boldsymbol{e}, \boldsymbol{\alpha})=J(\boldsymbol{w}, \boldsymbol{e})-\sum_{i=1}^{N} \alpha_{i}\left(\boldsymbol{w}^{T} \varphi\left(\boldsymbol{x}_{i}\right)+b+e_{i}-\boldsymbol{y}_{i}\right)
$$

where $\alpha$ is the Lagrange multiplier.

The conditions for optimality, obtained by partially differentiating Eq. 10 with respect to $w, b, e_{i}$, and $\alpha_{i}$ are given by:

$$
\left\{\begin{array}{l}
\frac{\partial L}{\partial \boldsymbol{w}}=0 \rightarrow \boldsymbol{w}=\sum_{i=1}^{m} \alpha_{i} \varphi\left(\boldsymbol{x}_{i}\right) \\
\frac{\partial L}{\partial b}=0 \rightarrow \sum_{i=1}^{m} \alpha_{i}=0 \\
\frac{\partial L}{\partial e_{i}}=0 \rightarrow \alpha_{i}=\gamma e_{i} \\
\frac{\partial L}{\partial \alpha_{i}}=0 \rightarrow w^{T} \varphi\left(\boldsymbol{x}_{i}\right)+b+e_{i}-y_{i}=0
\end{array}\right.
$$

From Eq. (11), the above optimization problem can be expressedin matrix form as:

$$
\left[\begin{array}{ll}
0 & \boldsymbol{I}^{T} \\
\boldsymbol{I} & \boldsymbol{\Omega}+\gamma^{-1} \boldsymbol{I}
\end{array}\right]\left[\begin{array}{l}
b \\
\boldsymbol{\alpha}
\end{array}\right]=\left[\begin{array}{l}
0 \\
\boldsymbol{y}
\end{array}\right]
$$

where

$$
\begin{aligned}
& \boldsymbol{y}=\left[y_{1}, y_{2}, \cdots y_{N}\right]^{T} \\
& \boldsymbol{\alpha}=\left[\alpha_{1}, \alpha_{2}, \cdots \alpha_{N}\right]^{T} \\
& \boldsymbol{\Omega}_{i j}=K\left(\boldsymbol{x}_{i}, \boldsymbol{x}_{j}\right)=\varphi\left(\boldsymbol{x}_{i}\right)^{T} \varphi\left(\boldsymbol{x}_{j}\right)
\end{aligned}
$$

and $\boldsymbol{I}$ is the identify matrix, $\boldsymbol{\Omega}$ is a square matrix, and $K\left(x_{i}, x_{j}\right)$ is a kernel function.

By solving Eq. (12), the estimated $\alpha$ and $b$ can be obtained. Finally, the resulting LS-SVM model can be described as:

$$
\boldsymbol{y}=\sum_{i=1}^{N} \alpha_{i} K\left(\boldsymbol{x}, \boldsymbol{x}_{i}\right)+b
$$


The most known kernel functions used in the literature are radial (Gaussian), polynomial, spline, or even sigmoidal functions [34]. In this study, a Gaussian radial basis kernel function which often gives a better result is used as the kernel function:

$$
K\left(\boldsymbol{x}, \boldsymbol{x}_{i}\right)=\exp \left(-\frac{1}{2 \sigma^{2}}\left\|\boldsymbol{x}-\boldsymbol{x}_{i}\right\|^{2}\right)
$$

where $\sigma$ is the kernel width parameter.

It is obvious that the regularization parameter $\gamma$ and the kernel width parameter $\sigma$ have a great effect on the performance of the LS-SVM model. Hence, an optimal regularization parameter $\gamma$ and kernel width parameter $\sigma$ should be selected to obtain the LS-SVM model with ideal predictive accuracy.

\subsection{LS-SVM modeling}

The training sample $\left(\boldsymbol{x}_{i}, \boldsymbol{y}_{i}\right)$ was constructed using the experimental data in Table 2, where the circumferential overlap, axial overlap and overscan number constituted the input vector $\boldsymbol{x}_{i}$, and the output vector $\boldsymbol{y}_{i}$ was the machined depth. The input and output dataset was conventionally normalized within the range $[0,1]$ before training. All input values were normalized during the prediction process using the following transformation formula [33]:

$$
\boldsymbol{u}_{\mathrm{nor}}=\frac{\boldsymbol{u}-\boldsymbol{u}_{\min }}{\boldsymbol{u}_{\max }-\boldsymbol{u}_{\min }}
$$

where $\boldsymbol{u}_{\text {nor }}$ is the normalized data, $\boldsymbol{u}$ is the initial input $\boldsymbol{x}_{i}$ or output $\boldsymbol{y}_{i}$, and $\boldsymbol{u}_{\min }$ and $\boldsymbol{u}_{\max }$ are the minimum and maximum values of input or output data.

The output values produced by the LS-SVM were not the actual values since all input values were normalized during the prediction process. The actual machined depth can be obtained from the output values of the LS-SVM model through inverse transform using Eq. (15).

As already mentioned in the previous section, adjusting the regularization parameter $\gamma$ and the kernel width parameter $\sigma$ can improve the predictive accuracy of the LS-SVM model. Grid search, most often used in the literature, is the simplest way to determine the values of these parameters [35]. In this research, the grid search and the leave-one-out cross-validation (LOO-CV) method [36] were used to determine the optimal regularization parameter $\gamma$ and kernel width parameter $\sigma$. For the LOO-CV method, $N-1$ random samples were taken out from all the $N$ samples each time, and the remaining one was used to assess training performance. The error of each assessment was set as the error, and then the root mean square error (RMSE) of $N$ samples was utilized as the modeling error as given by:

$$
R M S E=\sqrt{\frac{1}{N} \sum_{i=1}^{N} \text { error }_{i}^{2}}
$$


The performance of different parameters was evaluated by using the RMSE of LOO-CV as the objective function in the grid search.

A MATLAB Toolbox function was applied for the LS-SVM algorithm. In this study, the parameter ranges of LS-SVM were set to $\left[2^{-8}, 2^{11}\right]$ for the regularization parameter $\gamma$ and $\left[2^{-10}, 2^{8}\right]$ for kernel parameter $\sigma^{2}$, and the exponential jumping interval was set to 0.3 . By setting the upper and lower bounds for the regularization and kernel parameter and the jumping interval, the optimal sets of for parameters $\gamma$ and $\sigma^{2}$ leading to the smallest RMSE in this range were obtained. The grid search result is shown in Fig. 8. After grid searching, the optimum parameters are found as $\gamma=1910.85$ and $\sigma^{2}=7.46$ when $R M S E$ is equal to $0.031 \mu \mathrm{m}$.

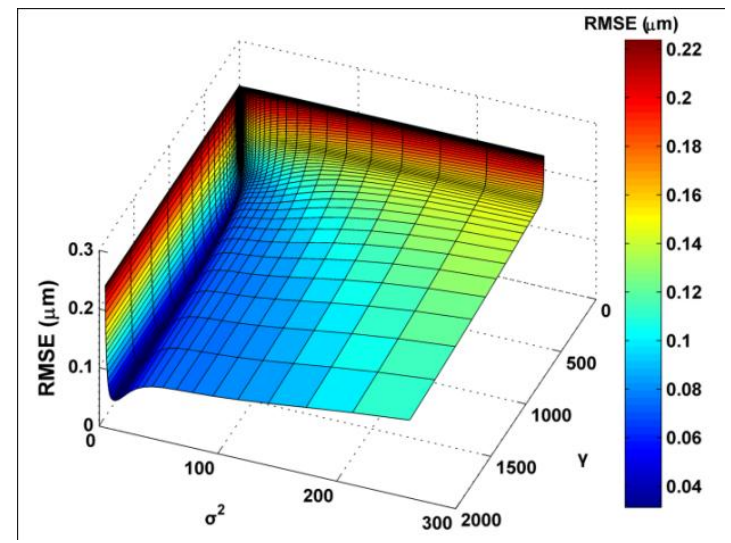

Fig. 8 Grid search result

The comparison of the predicted results obtained from the LS-SVM model using the optimal parameters and the experimental values are illustrated in Fig. 9. For the LS-SVM model, $R^{2}$ is 0.9999, $e_{\mathrm{R}}$ is $3.82 \%$ and $e_{\mathrm{M}}$ is $2.72 \%$. It is obvious that the LS-SVM model is of far better accuracy on training compared to the second-order regression model with $R^{2}$ of $0.9989, e_{M}$ of $59.26 \%$, and $e_{R}$ of $31.22 \%$.

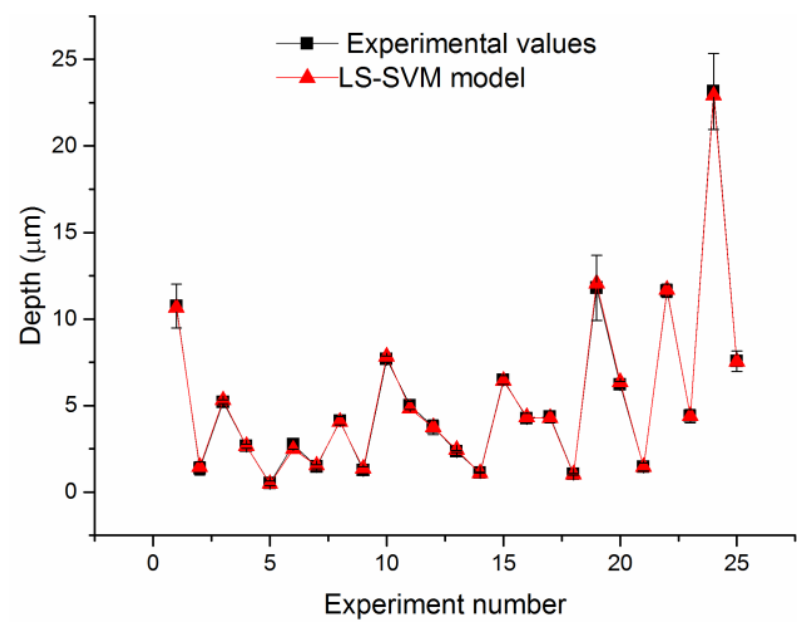

Fig.9 Comparison of experimental values and training results of regression model

\section{Model validation}




\subsection{Performance comparison}

To test the performance of the second-order regression and of the LS-SVM model, additional 5 experiments were conducted. For these experiments, the values of the laser process parameters were chosen within the range mentioned previously. The experiment data are given in Table 3.

The performance of the LS-SVM model was compared to that of the second-order regression model and both were validated against the actual experimental results, as shown in Fig. 10. The $R^{2}$ of second-order regression model is 0.9862 , the $e_{\mathrm{R}}$ is $31.73 \%$ and $e_{\mathrm{M}}$ is $24.49 \%$, while the $R^{2}, e_{\mathrm{R}}$ and $e_{\mathrm{M}}$ of the LS-SVM model are $0.9987,10.21 \%$ and $8.98 \%$, respectively. This indicates the comparative quality of performance of the LS-SVM model against the second-order regression model.

Table 3 Additional experimental data set

\begin{tabular}{ccccc}
\hline Exp no. & $\mathrm{A}(\%)$ & $\mathrm{B}(\%)$ & $\mathrm{C}$ & Depth (um) \\
\hline 1 & 0.600 & 0.900 & 5 & 4.72 \\
2 & 0.675 & 0.825 & 3 & 1.79 \\
3 & 0.750 & 0.600 & 9 & 4.38 \\
4 & 0.825 & 0.750 & 3 & 2.57 \\
5 & 0.900 & 0.675 & 1 & 1.35 \\
\hline
\end{tabular}

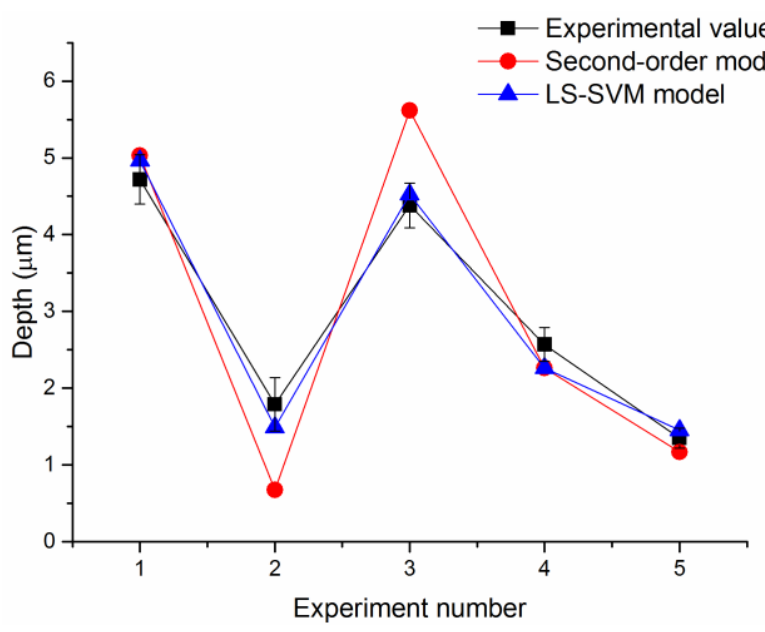

Fig.10 Comparison of experimental values and estimated results for the regression and LS-SVM models

\subsection{Case study}

Micro-channels with blended edges, instead of sharp edges, on medical needles are expected to reduce the edge effect and to reduce the insertion force. The cross-sectional views of micro-channels with sharp edges and blended edges are illustrated in Fig. 11.

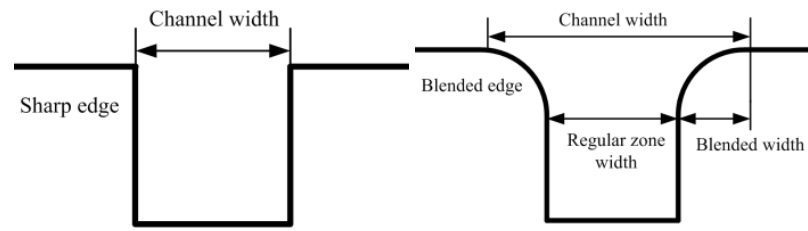

(a)

(b)

Fig. 11 Illustration of cross-sectional profiles of micro-channel with (a) sharp edges, (b) blended edges 
As a case of study, the creation of a channel with a length of $450 \mu \mathrm{m}$ and a width of $100 \mu \mathrm{m}$ (i.e., $50 \mu \mathrm{m}$ wide regular zone and $25 \mu \mathrm{m}$ wide blended zones) was modeled using the above-developed LS-SVM model. Then, an experiment was performed to assess the validity of the LS-SVM model. In this case, the blended edges were created by controlling the circumferential overlap of the laser pulse. Figure 12 shows the schematic of the machining of micro-channel with blended edges. For the left blended zone laser ablation began $25 \mu \mathrm{m}$ away from the left regular zone rim of the channel with the circumferential overlap linearly increasing from $65 \%$ to $90 \%$ as the beam moved toward the left regular zone rim. The circumferential overlap was set to $90 \%$ and kept constant in the regular textured zone. On the contrary, the $C O_{\mathrm{P}}$ was reduced linearly from $90 \%$ to $65 \%$ in the right blended zone. Laser scanning stopped at $25 \mu \mathrm{m}$ away from the right regular zone rim of the channel. During experimentation, the laser process parameters which have been kept constant were the axial overlap at $75 \%$, pulse energy at approximately $3 \mu \mathrm{J}$, and needle rotation speed at $10 \mathrm{rpm}$. The machining trajectory was repeated 3 times, i.e., the overscan number was 3. The machined micro-channel was measured at three different cross-section locations, and the results are shown in Fig. 13. Furthermore, the predicted cross-section geometry using the LS-SVM model is also shown in Fig. 13. As it can be seen, the predicted and experimental profiles are in good agreement within the width range of the channel (-50 to 50 micron meter along the "path length" axis in Fig.13). Outside of the width range of the channel, the predicted results show a slight deviation from the experimental values. The deviation is caused by the assumption made in the prediction calculations that the depth was assumed to be zero outside of the width range of the channel. Ideally, there is an abrupt change in the predicted depth profile at the two rims of the channel. However, in the practical machining process, the center of the first single-channel (laser beam with Gaussian energy overlapped) was set at the rim of the channel. Consequently, one half of the single-channel created a gradient curve profile outside of the width range. Hence, there is a discrepancy at the two rims of the channel between the predicted and experimental profiles.

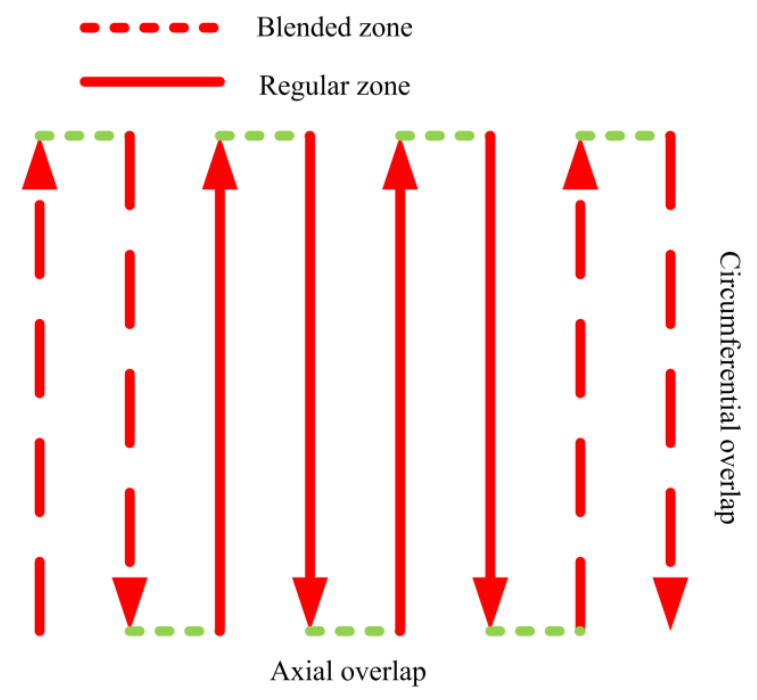

Fig. 12 Schematic of the machining of micro-channel with blended edges 


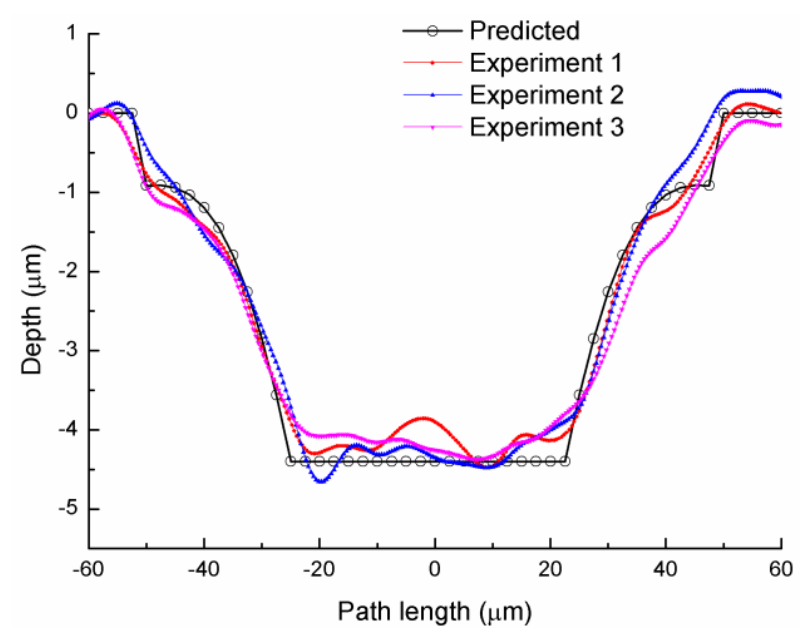

Fig 13 Comparison of experimental and predicted profiles

\section{Conclusions}

In this study, LST was applied to create micro-channels on medical needles. Controlling the circumferential overlap by two different methods, i.e., by changing the needle rotational speed and by changing the pulse frequency were first experimentally studied. The experimental results have shown that the machined depth increases with an increase in the circumferential overlap, whereas the needle rotational speed and pulse frequency have no obvious effect on the machined depth with the same circumferential overlap.

Considering three parameters, i.e., circumferential overlap, axial overlap and the overscan number, a second-order regression model for predicting machined depth was established based on Taguchi orthogonal experiments. It was shown that the regression model can predict the machined depth reasonably well. However, in order to improve predictive accuracy, a LS-SVM model for predicting the machined depth using the same experimental data was developed. It was found that the LS-SVM model was of a better accuracy on training compared to the second-order regression model. Additional 5 experiments were conducted to test the performance of the second-order regression and LS-SVM models. For the second-order regression prediction model, the $R^{2}$ was 0.99553 , the $e_{\mathrm{R}}$ was $22.51 \%$ and $e_{\mathrm{M}}$ was $17.28 \%$, whereas the $R^{2}, e_{\mathrm{R}}$ and $e_{\mathrm{M}}$ of LS-SVM model were $0.99887,10.68 \%$ and $8.96 \%$, respectively. As it was anticipated, the LS-SVM model provided better prediction capabilities because it generally offers the ability to model more complex nonlinearities than the second-order regression model can offer. A case study on micro-channels with blended edges was modeled using the developed LS-SVM model and experimentally tested. It was found that the predicted profile was in a good agreement with the experimental profiles. The LS-SVM model can be used to predict machined geometry of the micro-channels on medical needles.

\section{Acknowledgements}

This work was supported by National Science Foundation (Grant \#CMMI-0825722), Natural Science Foundation of Jiangsu Province (BK20150685) and the Korea Institute of Machinery and Materials. X. Wang also would like to acknowledge the Chinese Scholarship Council (CSC) for financial support. 


\section{References}

1. Han P, Che D, Pallav K, Ehmann KF. Models of the cutting edge geometry of medical needles with applications to needle design. Int J Mech Sci 2012;65:157-67.

2. Abolhassani N, Patel R, Moallem M. Needle Insertion into Soft Tissue: A Survey. Med Eng Phys 2007;29(4):413-31.

3. Chin KA, Perlas A, Chan VWS, Brull R. Needle visualization in ultrasound-guided regional anesthesia: challenges and solutions. Reg Anesth Pain Med. 2008;33(6):532-44.

4. Culp WC, McCowan TC, Goertzen TC, Habbe TG, Hummel MM, Leveen RF, Anderson JC. Relative ultrasonographic echogenicity of standard, dimpled, and polymeric-coated needles. J Vasc Interv Radiol 2000;11(3):351-8.

5. Deam RK, Kluger R, Barrington MJ, McCutcheon CA. Investigation of a new echogenic needle for use with ultrasound peripheral nerve blocks. Anesth Intensive Care 2007;35(4):582-6.

6. Hocking G, Hebard S. Echogenic technology can improve needle visibility during ultrasound-guided regional anesthesia. Reg Anesth Pain Med 2011;36(2):185-9.

7. Han P, Kim J, Ehmann KF, Cao J. Laser surface texturing of medical needles for friction control. Int J Mech Manuf Syst 2013;6(3):215-28.

8. Chae J, Park SS, Freiheit T. Investigation of micro-cutting operations. Int J Mach Tool Manu 2006;46(3-4):313-32.

9. Liu K, Lauwers B, Reynaerts D. Process capabilities of Micro-EDM and its applications. Int J Adv Manuf Tech 2010;47(1-4):11-9.

10. Rajurkar KP, Sundaram MM, Malshe AP. Review of electrochemical and electro discharge machining. Procedia CIRP 2013;6:13-6.

11. Mathew R, Sundaram MM. Modeling and fabrication of micro tools by pulsed electrochemical machining. J Mater Process Tech 2012;212(7):1567-72.

12. Hu X, Yu Z, Rajurkar KP. State-of-the-art review of micro ultrasonic machining, in: Proceedings of the ASME 2006 International Manufacturing Science and Engineering Conference, Ypsilanti, Michigan, USA, 2006, pp.1017-1024.

13. Allen DM, Shore P, Evans RW, Fanara C, O’Brien W, Marson S, O'Neil W. Ion beam, focused ion beam, and plasma discharge machining. CIRP Ann-Manuf Tech 2009;58(2):647-62.

14. Ali MY, Huang W, Fu Y. A review of focused ion beam sputtering. Int J Precis Eng Man 2010; 11(1):157-70.

15. Dubey AK, Yadava V. Laser beam machining-a review. Int J Mach Tool Manu 2008;48(6): 609-28.

16. Cheng J, Liu CS, Shang S, Liu D, Perrie W, Dearden G, Watkins K. A review of ultrafast laser materials micromachining. Opt Laser Technol 2013;46:88-102.

17. Guo P, Ehmann KF. Development of a tertiary motion generator for elliptical vibration texturing. Precis Eng 2013;37(2):364-71.

18. Singh R, Melkote SN. Characterization of a hybrid laser-assisted mechanical micromachining (LAMM) process for a difficult-to-machine material. Int J Mach Tool Manu 2007;47(7-8): $1139-50$

19. Zhang QH, Du R, Zhang JH, Zhang QB. An investigation of ultrasonic-assisted electrical discharge machining in gas. Int J Mach Tool Manu 2006;46(12-13):1582-8. 
20. Jiang C, Lau W, Yue T, Chiang L. On the maximum depth and profile of cut in pulsed Nd:YAG laser machining. CIRP Ann-Manuf Tech 1993;42(1):223-6.

21. Li Y, Latham WP, Kar A. Lumped parameter model for multimode laser cutting. Opt Laser Eng 2001;35(6):371-86.

22. Kim MJ. Transient evaporative laser-cutting with boundary element method. Appl Math Model 2000; 25(1):25-39.

23. Kim MJ. Transient evaporative laser cutting with moving laser by boundary element method. Appl Math Model 2004;28(10):891-910.

24. Yousef BF, Knopf GK, Bordatchev EV, Nikumb SK. Neural network modeling and analysis of the material removal process during laser machining. Int J Adv Manuf Tech 2003;22:41-53.

25. Tsai MJ, Li CH, Chen CC. Optimal laser-cutting parameters for QFN packages by utilizing artificial neural networks and genetic algorithm. J Mater Process Tech 208(1-3) (2008) 270-283.

26. Ciurana J, Arias G, Ozel T. Neural network modeling and particle swarm optimization (PSO) of process parameters in pulsed laser micromachining of hardened AISIH13 steel. Mater Manuf Process 2009;24(3):358-68.

27. Davis T, Cao J. Effect of laser pulse overlap on machined depth. Transaction of the North American Manufacturing Research Institution of SME 2010;38:291-8.

28. Gilbert D, Stoesslein M, Axinte D, Butler-Smith P, Kell J. A time based method for predicting the workpiece surface micro-topography under pulsed laser ablation. J Mater Process Tech 2014; 214(12):3011-88.

29. Sharma A, Yadava V. Modelling and optimization of cut quality during pulsed Nd:YAG laser cutting of thin Al-alloy sheet for straight profile. Opt Laser Technol 2012;44(1):159-68.

30. Wang X, Kang M, Fu X, Li C. Predictive modeling of surface roughness in lenses precision turning using regression and support vector machines. Int J Adv Manuf Tech 2013; doi:10.1007/s00170-013-5231-3

31. Suykens JAK, Vandewalle J. Least squares support vector machine classifiers. Neural Process Lett 1999;9(3):293-300.

32. Salgado DR, Alonso FJ, Cambero I, Marcelo A. In-process surface roughness prediction system using cutting vibrations in turning. Int J Adv Manuf Tech 2009;43(1-2):40-51.

33. Xu WJ, Wei ZF, Sun J, Wei L, Yu ZY. Surface quality prediction and processing parameter determination in electrochemical mechanical polishing of bearing rollers. Int J Adv Manuf Tech 2012;63(1-4):129-36.

34. Zhang LX, Jia ZY, Wang FJ, Liu W. A hybrid model using supporting vector machine and multi-objective genetic algorithm for processing parameters optimization in micro-EDM. Int $\mathbf{J}$ Adv Manuf Tech 2010;51(5-8):575-86.

35. Caydas U, Ekici S. Support vector machines models for surface roughness predictionin CNC turning of AISI 304 austenitic stainless steel. Int J Adv Manuf Tech 2012;23(3):639-50.

36. Dong M, Wang N. Adaptive network-based fuzzy inference system with leave-one-out cross-validation approach for prediction of surface roughness. Appl Math Model 2011;35(3): $1024-35$. 